\title{
GRAHAM-LITTLE-PICCARDI-LASSUEUR SYNDROME: TWO CASE REPORTS AND REVIEW OF THE LITERATURE
}

\author{
L. Dourmishev', N. Mironova', I. Popov', D. Rusinova ${ }^{3}$, M. Balabanova', L. Miteva ${ }^{1}$ \\ ${ }^{1}$ Department of Dermatology and Venerology, Medical University - Sofia, Bulgaria \\ ¿University Hospital "N. I. Pirogov" - Sofia, Bulgaria \\ ${ }^{3}$ MBAL "Tota Venkova" - Gabrovo, Bulgaria
}

\begin{abstract}
Graham-Little-Piccardi-Lassueur syndrome (GLPLS) is a rare syndrome characterized by the triad of cicatricial alopecia of the scalp, non-cicatricial alopecia of the axilla and groin and follicular lichen planus eruptions on the trunk and extremities. GLPLS is considered to be a variant of lichen planopilaris. We report two cases that have fulfilled all of the criteria for GLPLS. The first case was a 71-year-old woman, admitted to the Department of Dermatology for pruritic perifollicullar erythema and scaling of the scalp, cicatricial scalp alopecia and hair loss of the axilla and pubic region for five months. Subsequently, follicular hyperkeratotic eruptions and hyperpigmented macules on the skin of the chest and abdomen appeared. The second case was a 48-year-old man with pruritic follicular papules on the face, trunk and extremities for four months. All of the laboratory examinations in both patients were within normal limits. No alternation in the general condition of the patients was observed. Histological examinations in both patients confirmed the diagnosis GLPLS. The patients were treated with systemic and local corticosteroid resulting in marked improvement of the skin lesions; however, cicatricial scalp alopecia showed no response to the treatment.
\end{abstract}

Key words: lichen planopilaris, cicatricial alopecia, Graham-Little-Piccardi-Lassueur syndrome

Corresponding author: Lyubomir Dourmishev, MD, PhD, Department of Dermatology and Venerology, Medical University, 1431 Sofia, Bulgaria, e-mail:__dourmishev@yahoo.com

\section{INTRODUCTION}

raham-Little-Piccardi-Lassueur

syndrome (GLPLS) is a rare lichenoid dermatosis characterized by cicatricial alopecia of the scalp; non-cicatricial alopecia of the axilla and pubic area and disseminated spinous or acuminated follicular papules distributed on the trunk and extremities [1]. Most of the reported cases are in middle aged women. Only a few cases have been reported in the literature wherein the disease has affected males.
GLPLS is considered to be a variant of lichen planopilaris (LPP) of the body and scalp. About half of the patients with GLPLS have experienced at least one episode of typical oral or cutaneous lichen planus [2]. The disease is sporadic and current evidence shows no genetic predisposition. Only one case of familial GLPLS was reported from Viglizzo G et al. in 2004 in a mother and her daughter and both patients had HLA-DR1 genotype [3]. GLPLS is rarely associated with other diseases, one case which developed after hepatitis B vaccination [4] and another one of a 
female patient with complete androgen insensitivity syndrome have been reported [5].

We report two cases that have fulfilled all of the criteria for GLPLS.

\section{CASE REPORT 1}

A 71-year-old woman was admitted to our department for evaluation of pruritic lesions on the scalp for five months and pruritic eruption over trunk which appeared two weeks before her admission. She was treated unsuccessfully with $2 \%$ ketoconazole shampoo and betamethasone dipropionate/salicylic acid lotion for the scalp lesions.

The clinical examination revealed hyperkeratotic follicular papules, perifollicullar erythema and atrophic alopecia on parietal region of the scalp (Fig.1), likewise follicular eruptions and hyperpigmented macules on the skin of the chest and abdomen (Fig. 2).
In the axilla and pubic area thinning and hair loss has been noted. The mucous membranes were intact while the finger nails revealed longitudinal ridging and yellow discoloration.

All of the blood tests were in normal ranges. Histological examination of the follicular eruptions of the skin disclosed hyperkeratosis, epidermal atrophy, hydropic degeneration of the basal cell layer and band-like lymphocytic infiltration in the upper dermis (Fig. 3). In addition, the histological examination of the scalp lesions revealed hydropic degeneration of the basal cell layer of the follicles and perifollicular lymphocytic infiltration that suggested histological findings of cicatricial alopecia of the scalp.

A treatment with methylprednisolone $40 \mathrm{mg} /$ daily with stepwise decreasing doses and topical flucinolone acetonid was initiated. Two months later the skin lesions moderately improved, however cicatricial scalp alopecia did not respond to the treatment.

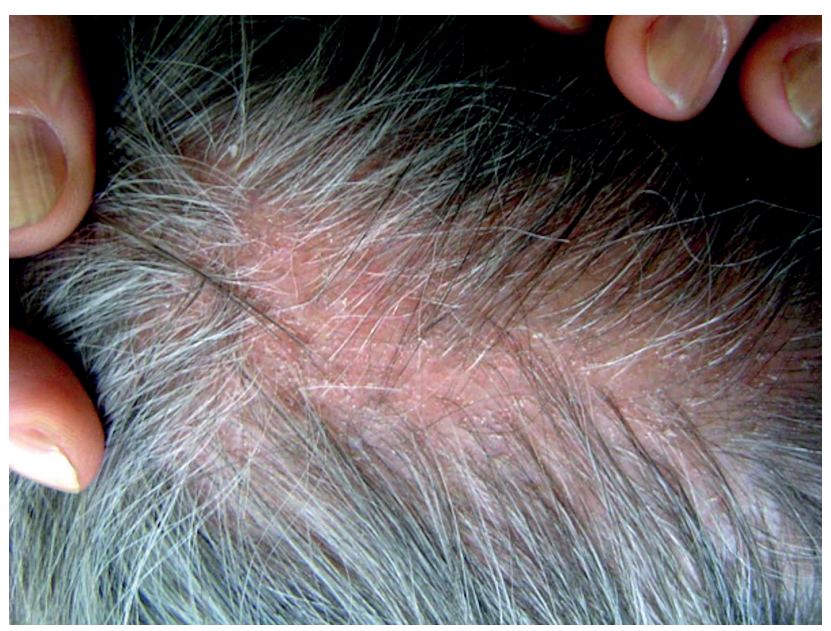

Fig. 1. Hyperkeratotic follicular papules, perifollicular erythema and atrophic alopecia on the parietal region of the scalp

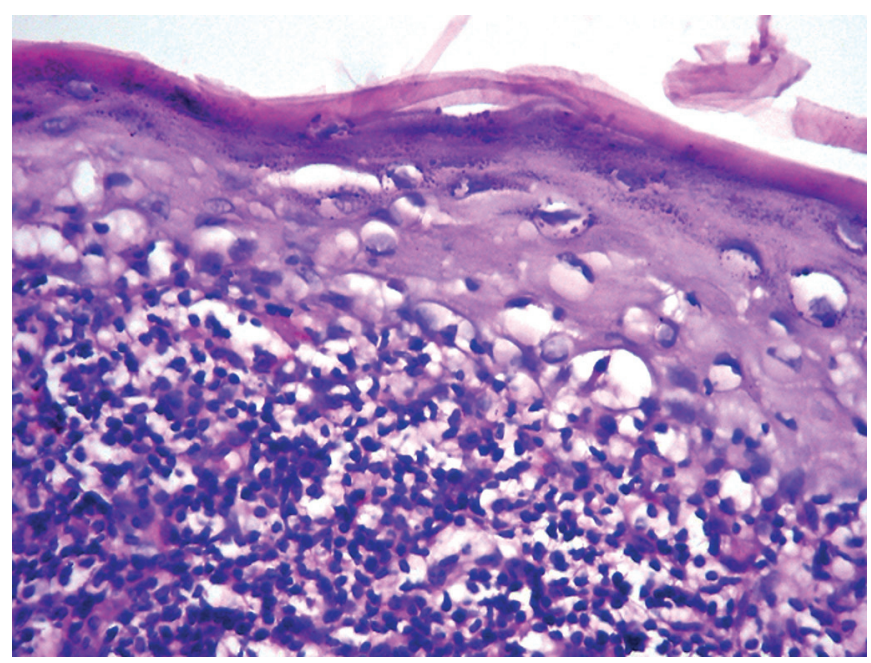

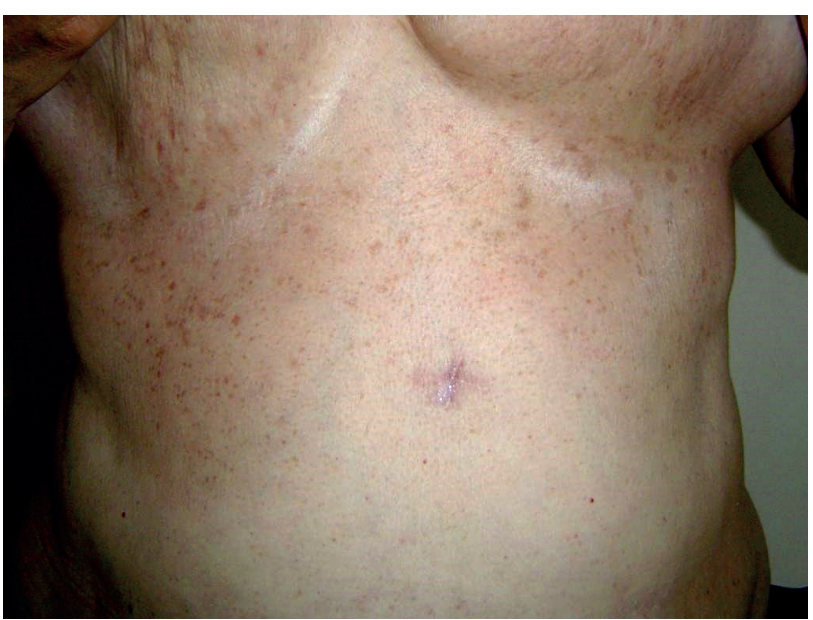

Fig. 2. Follicular papules and hyperpigmented macules on the skin of the lower chest and abdomen

Fig. 3. Histology of the skin lesions reveal hydropic degeneration of the basal cell layer of the follicles and perifollicular lymphocytic infiltration (H\&E x 400) 


\section{CASE REPORT 2}

A 48-year-old man was accepted to the Department of Dermatology for a pruritic follicular papule on the face, trunk and extremities lasting for four months. He was treated with topical clobetasol propionate with a good result. The patient had ischemic stroke in his past medical history. No alternation in the general condition of the patient was observed. The clinical examination revealed follicular papules and hyperpigmented macules on the skin of trunk (Fig. 4) and arms. There was alopecia on frontal, temporal and parietal regions of the scalp. Thinning of the hair and hair loss without scar formation were noted in the axilla and pubic areas.

The results of routine laboratory examinations including complete blood count, serum biochemistry, urine analysis and liver function tests were within normal ranges. Histological examination of the follicular papule on the arm revealed hyperkeratosis, hydropic degeneration of the basal cell layer and lymphocytic infiltration of the papillary dermis.

We started treatment with dexamethsone $8 \mathrm{mg} /$ daily in gradually decreasing doses and local treatment with flucinolone acetonid cream. The follicular rash on the trunk showed moderate improvement after one-month treatment except the cicatricial scalp alopecia which remained unaffected.

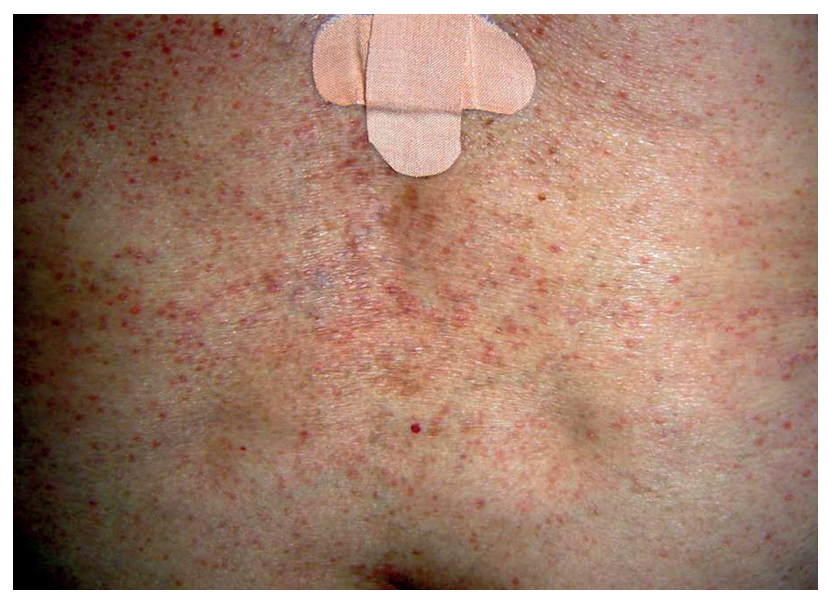

Fig. 4. Lichenoid papules on the skin of trunk

\section{DISCUSSION}

In 1914 Piccardi described the first case of progressive cicatricial alopecia of the scalp, non-cicatricial alopecia of the axilla and pubic area and follicular lichen planus on the trunk and extremities named as "keratotic spinulosa". In 1915 Graham-Little [6] published a similar case referred by Lassueur, which was entitled as "folliculitis decalvans et atrophicans".
Subsequently, sporadic cases have been reported in the literature and the majority of them were of middle aged Caucasian women like our first patient. Almost all reported cases presented with progressive cicatricial alopecia of the scalp, non-cicatricial alopecia of the axilla and pubic area and follicular papules of the body and extremities [1]. Less commonly the hair follicle's lesions can develop on the eyebrows and sides of face. Sometimes individuals with GLPLS experience itching around affected areas. In addition to the triad, a positive pull test for anagen hair can be present. Generally, the earliest change has been patchy cicatricial alopecia of the scalp that precedes the follicular lichen planus eruptions by months or years [7]. Progressive cicatricial scalp alopecia leading to permanent hair loss and may produce psychosocial distress for the patients. Cicatricial alopecia of the scalp is chronic and progresses through perifollicular erythema, follicular hyperkeratosis, patches of cicatricial alopecia with tufts of normal hair follicles; and finally, cicatricial alopecia with permanent hair loss, comparable to pseudopelade of Brocq [1].

Scalp affection of GLPLS is considered to be one of the 3 clinical variants of lichen planopilaris (LPP). Other variants include follicular lichen planus, and frontal fibrosing alopecia [8]. Recently, fibrosing alopecia in a pattern distribution has gained some popularity as a fourth variant of LPP [9].

In our first patient cicatricial scalp alopecia initiated four months before the appearance of the skin eruptions with perifollicular erythema, follicular hyperkeratosis and later small patches of cicatricial alopecia appeared. Histological findings of cicatricial scalp alopecia in the early stage represent inflammatory perifollicular lichenoid infiltration and is often associated with the bulge region of the follicle. Bulge region contains the stem cell responsible for regenerating about two thirds of the hair follicle which is nonpermanent [10]. Inflammation in this area could lead to cicatricial alopecia [10]. In the final stage of GLPLS atrophic epidermis, sclerosis in derma, keratinous follicular plugs and empty hair shaft can be observed.

Direct immunofluorescence (DIF) studies have reported colloid bodies and a linear band of fibrin and/ or fibrinogen along the dermo-epidermal junction [11]. A DIF study of LPP patients discovered IgG deposits in $7 \%$ of cases, $\lg M-$ in $45 \%, \lg A-$ in $19.3 \%$, and $\mathrm{C} 3$ in $28.1 \%$ [12].

The presence of non-cicatricial alopecia of the axilla, pubic area and occasionally eyebrows are clinically obvious and skin biopsy is not necessary for confirmation. Histological findings include inflammatory cell infiltration and follicular damage, however the 
hair loss is not permanent [11]. In our patients the hair loss of the axillary and pubic area was associated with thinning of the residual hairs, by without scaring.

Follicular lichen planus eruptions on the trunk, extremities and occasionally face, may be associated with typical oral involvement of lichen planus. It might be developed rapidly over a period of weeks or months. A skin biopsy reveals hyperkeratosis, acanthosis, hypergranulosis with occasional "saw-toothed rete ridges", lymphocytic infiltration in upper dermis and dyskeratotic keratinocytes [7].

Etiology of GLPLS is unknown but several hypotheses have been proposed. Based on immunologic hypothesis, HLA-antigens enhance a T-cell mediated immune response. HLA-DR is one of the several HLA genes associated with lichen planus and GLPLS [3]. Furthermore, familial GLPL was described in a mother and daughter, though the association was uncertain. The viral hypothesis is supposed, because both lichen planus and GLPLS have been reported after $\mathrm{HBV}$ vaccination and $\mathrm{HBV}$ vaccine might trigger lichen like eruption. Lichen planus-like eruption have not been reported after other vaccinations [13]. A case report of a 19-years old male GLPLS patient with testicular feminization supports the possibility that hormonal disfunction plays role in disease pathogenesis [5]. Likewise, altered hormone level have been suggested because most of the GLPLS patients are premenopausal and postmenopausal women, however rare cases in a child and a teenager have been reported as well [14]. Other suspected etiologies are neuropsychological stress and vitamin A deficiency [15]. A case series of 29 patients with LPP and its variants, including GLPL, revealed a thyroid disorder in $24 \%$ of patients, the most prevalent of which was Hashimoto thyroiditis [16]. Other case-control study by Mesinkovska et al. [17] also found a considerable increase in the prevalence of thyroid gland disease among patients with LPP vs controls. Hence it might have a strong association between the comorbidities of thyroid dysfunction and GLPL [17-19].

Rodríguez-Bayona $B$ et al. proposed an autoimmune theory of the disease and stated that the major autoantigen in a GLPL syndrome patient was INCENP protein [20]. INCENP is a major component of the centromere during several phases of the mitotic cell cycle where it plays an essential role in chromosome segregation and participates in the mechanisms regulating mitosis [21]. The major centromeric autoantigens are recognized as targets for other autoimmune diseases as CREST syndrome and systemic sclerosis [22]. Another interesting finding in the pathogenesis of GLPLS is an altered distribution of integrins in follicular keratinocytes in follicular lichen planus areas [23]. Integrin a6 subunit was present with a basolateral distribution, the $\beta 4$ subunit showed discontinuous expression at the basal pole while in uninvolved skin of the scalp the distribution of $\alpha 6 \beta 4$ was exclusively basal [23]. Altered integrin expression, which was shown in active LPP lesions, could explain the phenomenon of anagen hair on pull test [2].

The differential diagnosis of GLPL includes other variants of LPP as well as discoid lupus erythematous (DLE), pseudopelade of Brocq, pityriasis rubra pilaris, acne keloidalis, central centrifugal scarring alopecia, follicular mucinosis, and folliculitis decalvans, which also needs to be ruled out. The differentiation between LPP and DLE is hard. It includes lack of central erythema and telangiectasias within the lesions. Histologically, the lymphocytic dermatitis and folliculitis can be indistinguishable, but subtle findings suggesting DLE may be present, such as increased mucin in the reticular dermis, a focally thinned epidermis, and less severe dermal sclerosis when compared to cases of LPP [2]. Pseudopelade of Brocq is an end-stage of hair loss in LPP rather than a separate condition. It is considered to be the end point of GLPL as well as DLE and others when the inflammation has subsided and the cicatricial alopecia is stable. For the duration of active disease, GLPL is classified as an unstable cicatricial alopecia that has a tendency to progress and recur periodically [24]. Folliculitis decalvans can also mimic GLPL during a period when the pustules have resolved; however, a neutrophilic infiltrate will be present in histology.

No standard treatments have been introduced for GLPLS and patients tend to be treated empirically for this condition. In recent years, cyclosporine has been the most effective for the treatment of patients with GLPLS [25]. The dose of $4 \mathrm{mg} / \mathrm{kg} /$ day for 3 months shows significant decrease in follicular papules, attenuation of hair loss, and patchy hair regrowth up to complete healing. The other alternatives are methotrexate, showing improvement in $85 \%$ of patients and mycophenolate mofetil [26]. Scalp and cutaneous lesions are successfully treated by thalidomide [27, 28]. Moreover, thalidomide has been found effective in the therapy of cicatricial alopecia due discoid lupus erythematosus [28].

Topical, intralesional, and systemic corticosteroids; retinoids; antimalarials; tetracycline antibiotics; metronidazole; pioglitazone; PUVA therapy have shown limited success according to some authors [25]. However, in our cases we achieved a regression of the follicular lesions with combination of systemic and local corticosteroids, although scaring hair loss did not recover. The aim of treatment is to stop pro- 
gression, prevent further alopecia, and symptomatic management.

\section{CONCLUSION}

In conclusion we present two patients, that have fulfilled all of the criteria for GLPLS. The disease was successfully controlled with systemic and local steroids, nevertheless the cicatricial alopecia remained unaffected.

Disclosure summary: The authors have nothing to disclose.

\section{REFERENCES}

1. Zegarska B, Kallas D, Schwartz RA, et al. Graham-Little syndrome. Acta Dermatovenerol Alp Pannonica Adriat. 2010;19(3):39-42.

2. Assouly P, Reygagne P. Lichen planopilaris: Update on diagnosis and treatment. Semin Cutan Med Surg. 2009;28(1):3-10.

3. Viglizzo G, Verrini A, Rongioletti F. Familial Lassueur-GrahamLittle-Piccardi syndrome. Dermatology. 2004;208(2):142-4.

4. Meinhard J, Stroux A, Lünnemann L, et al. Lichen planopilaris: Epidemiology and prevalence of subtypes - a retrospective analysis in 104 patients. J Dtsch Dermatol Ges. 2014; 12(3):229-35, 229-36.

5. Vega Gutiérrez J, Miranda-Romero A, Pérez Milán F, et al. Graham Little-Piccardi-Lassueur syndrome associated with androgen insensitivity syndrome (testicular feminization). J Eur Acad Dermatol Venereol. 2004;18(4):463-6.

6. Graham-Little EG. Folliculitis decalvans et atrophicans. $\mathrm{Br} \mathrm{J}$ Dermatol. 1915;27: 183-5.

7. Bardazzi F, Landi C, Orlandi C, et al. Graham Little-PiccardiLassueur syndrome following HBV vaccination. Acta Derm Venereol. 1999;79(1): 93.

8. Kang $\mathrm{H}$, Alzolibani AA, Otberg $\mathrm{N}$, et al. Lichen planopilaris. Dermatol Ther. 2008;21(4):249-256.

9. Ramanauskaite A, Trüeb RM. Facial Papules in Fibrosing Alopecia in a Pattern Distribution (Cicatricial Pattern Hair Loss). Int J Trichology. 2015;7(3):119-122.

10. Sehgal VN, Srivastva G, Bajaj P. Cicatricial (scarring) alopecia. Int J Dermatol. 2001;40(4):241-248.

11. Chieregato C, Zini A, Barba A, et al. Lichen planopilaris: report of 30 cases and review of the literature. Int J Dermatol. 2003;42(5):342-5.

12. Agarwal R, Singh M, Dahiya R, et al. Direct immunofluorescence findings in cicatricial alopecia: A retrospective study of 155 cases. Indian J Pathol Microbiol. 2019;62(1):103-106.
13. Rübsam K, Schroll A, Weisenseel $P$ et al. Lichen planus and hepatitis virus infections: causal association? J Dtsch Dermatol Ges 2011;9(6):464- 8.

14. Chieregato C, Zini A, Barba A, et al. Lichen planopilaris: report of 30 cases and review of the literature. Int J Dermatol. 2003;42:342-345.

15. Alirezaei P, Ahmadpanah M, Rezanejad A, et al. Compared to Controls, Individuals with Lichen Planopilaris Have More Depression, a Lower Self-Esteem, and a Lower Quality of Life. Neuropsychobiology. 2019;78(2):95-103.

16. Cevasco NC, Bergfeld WF, Remzi BK, et al. A case-series of 29 patients with lichen planopilaris: the Cleveland Clinic Foundation experience on evaluation, diagnosis, and treatment. J Am Acad Dermatol. 2007;57(1):47-53.

17. Mesinkovska NA, Brankov N, Piliang M, et al. Association of lichen planopilaris with thyroid disease: a retrospective casecontrol study. J Am Acad Dermatol. 2014;70(5):889-892.

18. Vashi N, Newlove T, Chu J, et al. Graham-Little-Piccardi-Lassueur syndrome. Dermatol Online J. 2011;17(10):30.

19. Mardones F, Shapiro J. Lichen planopilaris in a Latin American (Chilean) population: demographics, clinical profile and treatment experience. Clin Exp Dermatol. 2017;42(7):755759.

20. Rodríguez-Bayona B, Ruchaud S, Rodríguez C, et al. Autoantibodies against the chromosomal passenger protein INCENP found in a patient with Graham Little-Piccardi-Lassueur syndrome. J Autoimmune Dis. 2007;4:1. doi: 10.1186/17402557-4-1.

21. Adams RR, Carmena M, Earnshaw WC. Chromosomal passengers and the (aurora) ABCs of mitosis. Trends Cell Biol. 2001;11(2):49-54.

22. Hamdouch K, Rodríguez C, Pérez-Venegas J, et al. AntiCENPI autoantibodies in scleroderma patients with features of autoimmune liver diseases. Clin Chim Acta. 2011;412(2324):2267-71.

23. D`Ovidio R, Sgarra C, Conserva A, et al. Alternated integrin expression in lichen planopilaris. Head Face Med. 2007; 3:11.

24. Dogra S, Sarangal R. What's new in cicatricial alopecia? Indian J Dermatol Venereol Leprol. 2013;79(5):576-590.

25. Bianchi L, Paro Vidolin A, Piemonte P, et al. Graham LittlePiccardi-Lassueur syndrome: effective treatment with cyclosporin A. Clin Exp Dermatol. 2001;26(6):518-20.

26. Babahosseini H, Tavakolpour S, Mahmoudi H, et al. Lichen planopilaris: retrospective study on the characteristics and treatment of 291 patients. J Dermatolog Treat. 2019;30(6):598-604.

27. Boyd AS, King LE Jr. Thalidomide-induced remission of lichen planopilaris. J Am Acad Dermatol. 2002;47(6):967-8.

28. Dourmishev A, Serafimova D, Marina S, et al. Thalidomide used in the therapy of aphthous lesions in Behcet?s disease and skin changes in lupus erythematosus. Dermatol. and Venereol. (Sofia), 2000;39(1):16-18. [Article in Bulgarian].

Received: 08 July 2020, Accepted: 15 July 2020 